\title{
Rap1b is required for normal platelet function and hemostasis in mice
}

\author{
Magdalena Chrzanowska-Wodnicka, ${ }^{1}$ Susan S. Smyth, ${ }^{1}$ Simone M. Schoenwaelder, ${ }^{2}$ \\ Thomas H. Fischer, ${ }^{3}$ and Gilbert C. White II
}

\begin{abstract}
1Department of Medicine and Carolina Cardiovascular Biology Center, University of North Carolina at Chapel Hill, Chapel Hill, North Carolina, USA.
${ }^{2}$ Australian Centre for Blood Diseases, Monash University, Alfred Medical Research and Education Precinct, Prahran, Victoria, Australia. ${ }^{3}$ Francis Owen Blood Research Laboratory, Department of Pathology and Laboratory Medicine, University of North Carolina at Chapel Hill, Chapel Hill, North Carolina, USA.
\end{abstract}

\begin{abstract}
Rap1b, an abundant small GTPase in platelets, becomes rapidly activated upon stimulation with agonists. Though it has been implicated to act downstream from G protein-coupled receptors (GPCRs) and upstream of integrin $\alpha_{\mathrm{IIb}} \beta_{3}$, the precise role of Rap1b in platelet function has been elusive. Here we report the generation of a murine rap1b knockout and show that Rap1b deficiency results in a bleeding defect due to defective platelet function. Aggregation of Rap1b-null platelets is reduced in response to stimulation with both GPCR-linked and GPCR-independent agonists. Underlying the defective Rap1b-null platelet function is decreased activation of integrin $\alpha_{\mathrm{II}} \beta_{3}$ in response to stimulation with agonists and signaling downstream from the integrin $\alpha_{\text {IIb }} \beta_{3}$. In vivo, Rap1b-null mice are protected from arterial thrombosis. These data provide genetic evidence that Rap1b is involved in a common pathway of integrin activation, is required for normal hemostasis in vivo, and may be a clinically relevant antithrombotic therapy target.
\end{abstract}

\section{Introduction}

Rap proteins are small GTPases from the Ras family, and genes encoding them are highly conserved across species (1-4). Genetic analysis of Rap 1 function in lower eukaryotes has revealed that Rap is critical for development, as loss-of-function mutations are lethal in Drosophila (3) and Dictyostelium (5). In mammals, the Rap family consists of 2 rap 1 genes, rap1a and rap1b, encoding proteins that are $95 \%$ identical, and 2 rap 2 genes encoding proteins that are $65 \%$ homologous with the Rap1 proteins. Rap1 shares a high degree of homology with Ras, especially in the effector region, and initial observations that Rap1 reverted the Ras-transformed phenotype in fibroblasts (6) and Xenopus oocytes (7) prompted investigations of the role of Rap as a Ras antagonist. While the Ras antagonist function of Rap remains controversial, there is increasing evidence that Rap signaling is involved in regulation of multiple cellular processes, including cell differentiation and adhesion (8-11).

While Rap1 proteins are ubiquitously expressed in tissues, Rap1b is the predominant Rap1 isoform and the most abundant Ras family member in platelets (12), where it becomes rapidly activated upon stimulation with a variety of agonists acting through distinct signaling pathways $(13,14)$. Stimulation of the $\mathrm{G \alpha}_{\mathrm{q}}$-linked PAR4 activates phospholipase $\mathrm{C} \beta$ (PLC $\beta$ ), leading to phosphoinositol hydrolysis and an intracellular calcium increase, providing a necessary and sufficient stimulus for Rap1b activation (13), which is later sustained by activated protein kinase C (15). ADP stimulates Rap1b activation via a calcium-independent pathway downstream from its $\mathrm{G \alpha}_{\mathrm{i}}$-coupled $\mathrm{P} 2 \mathrm{Y}_{12}$ receptor and is dependent on activation of phosphoinositide 3 -kinase $(16,17)$ and its lipid product phosphatidylinositol 3,4,5-triphosphate (18).

Nonstandard abbreviations used: GPCR, G protein-coupled receptor; GPVI, glycoprotein GPVI; PKA, cAMP-dependent protein kinase; PLC $\beta$, phospholipase C $\beta$; PRP, platelet-rich plasma; VASP, vasodilator-stimulated phosphoprotein.

Conflict of interest: The authors have declared that no conflict of interest exists.

Citation for this article: J. Clin. Invest. 115:680-687 (2005)

doi:10.1172/JCI200522973
While Rap1b activation is a common feature of platelet activation, the role it plays in platelets is not well understood. The current understanding of Rap $1 \mathrm{~b}$ function has been derived from overexpression studies in cultured cells involving Rap1-activating guanine exchange factors, dominant active forms of Rap1, or GTPase-activating proteins, which accelerate the low intrinsic GTPase activity of Rap, leading to its inactivation. Such studies in megakaryocytes, the precursors of platelets, and in a megakaryoblastic cell line have shown a correlation between the conditions that promote the activated state of Rap $1 \mathrm{~b}$ and enhanced agonistinduced activation of the platelet-specific integrin $\alpha_{\mathrm{IIb}} \beta_{3}(19-21)$. This integrin plays a central role in hemostasis, and defects in its activation lead to platelet aggregation deficiency and bleeding. Severe examples of such defects are present in Glanzmann thrombasthenia platelets, which lack functional integrin $\alpha_{\mathrm{IIb}} \beta_{3}$, and in mouse integrin $\beta_{3}$ subunit knockouts, which have a severe bleeding disorder and reduced survival (22). To address the exact role Rap1b plays in vivo, both at the organism level and in platelets, which are not amenable to genetic manipulation, we created and characterized the phenotype of Rap1b-deficient mice.

\section{Results}

Generation of Rap1b-deficient mice. Rap1b-null mice were created by replacing the entire coding sequence of the murine rap $1 b$ gene with a neomycin-resistance gene (Figure 1). Of the first 91 mice generated from the rap $1 b^{+/-}$intercrosses, at weaning, $42.9 \%$ were $r a p 1 b^{+/+}$, $52.7 \%$ were rap $1 b^{+/-}$, and $4.4 \%$ were rap $1 b^{-/-}$, indicating $85 \%$ embryonic and perinatal lethality of the Rap1b-null mice. Analysis of staged embryos derived from heterozygous intercrosses revealed that at least $40 \%$ of $\mathrm{rap}^{1 b^{-/}}$embryos had abdominal, cranial, and hepatic bleeding (Figure 1F) and died in utero between E13.5 and E18.5, while the remaining deaths occurred perinatally.

Surviving Rap1b-null mice were smaller than their littermates but appeared otherwise normal, with no overt cancer or hematological defects and normal life spans. Rap1b-null mice were fertile and produced $45 \%$ smaller litters at weaning compared with wild-type mice 
A
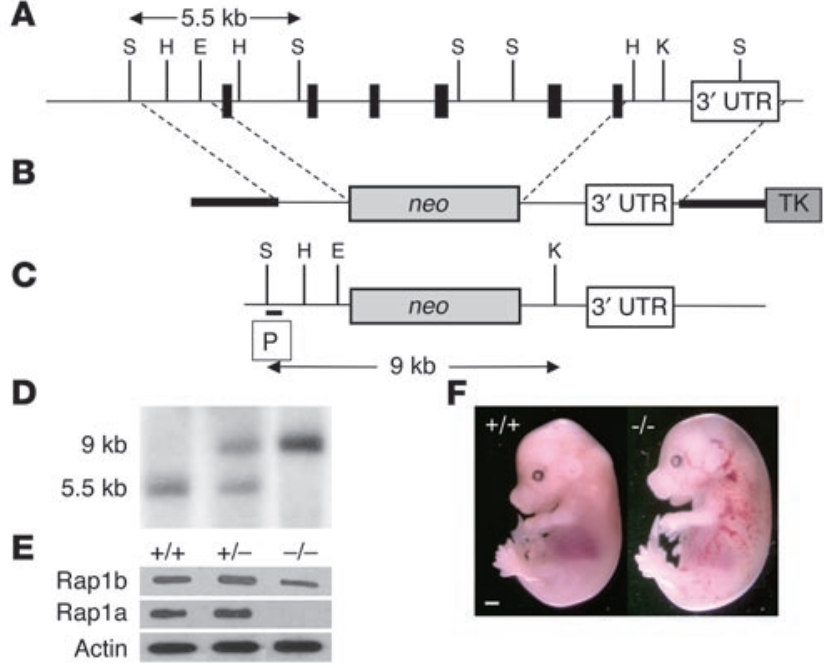

Figure 1

Targeted inactivation of the rap $1 b$ gene. (A) The murine rap $1 b$ gene consists of 6 coding (bands) and 1 untranslated exon (3' UTR, open box). (B) The targeting vector contains $7.8 \mathrm{~kb}$ of genomic DNA flanking the neomycin-resistance cassette (neo). TK, thymidine kinase. (C) After homologous recombination, the neo cassette replaces the complete coding sequence of the rap1b gene. (D) Southern blot analysis of mouse tail DNA from heterozygous intercrosses digested with Ssp/ and $\mathrm{Kpnl}$ using a probe $(\mathrm{P})$ that detects $5.5-\mathrm{kb}$ and $9-\mathrm{kb}$ fragments in the wild-type and knockout allele, respectively. (E) Western blot analysis of protein expression in platelets of indicated genotype. (F) Morphology of E15.5 wild-type (+/+) and Rap1b-null (-/-) embryos. Scale bar: $1 \mathrm{~mm}$. E, EcoRl; H, HinDIII; K, Kpnl; S, Sspl.

of the same genetic background ( $4.0 \pm 1.75$ vs. $7.2 \pm 2.36$ pups, respectively; mean $\pm \mathrm{SD}, n=40$ ). This slightly higher survival rate of Rap1bnull mice in homozygous intercrosses compared with heterozygous intercrosses may have been caused by a genotypic or phenotypic compensation that has ameliorated the consequences of Rap $1 \mathrm{~b}$ loss. Alternatively, larger litter size in $\mathrm{rap}^{1 \mathrm{~b}^{-1}}$ intercrosses could have resulted from a more uniform size of embryos and smaller likelihood of embryos being discarded by the mother.

Tail bleeding time is increased in Rap1b-null mice and in Rap1b-null bone marrow transplants. Rap1b-null mice had normal platelet counts and did not exhibit evidence of spontaneous bleeding. To evaluate the effect of Rap1b deficiency on hemostasis, we compared tail bleeding times of Rap1b-null mice and normal mice (Figure 2). The median time to bleeding cessation was significantly prolonged $(P<0.0001)$ in Rap1b-null mice compared with normal mice of the same genetic background $(460.4 \pm 35.3$ seconds vs. $101.1 \pm 13.2$ seconds, respectively; mean \pm SEM).

To determine whether abnormal platelet function was sufficient to cause the bleeding time prolongation observed in Rap1b-null mice, or whether other factors, such as defective vasculature, were involved, we reconstituted lethally irradiated wild-type mice with bone marrow harvested from Rap1b-null mice to create chimeric

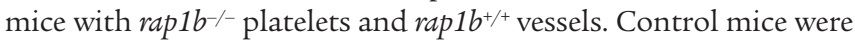
irradiated and rescued with bone marrow from wild-type mice. Fourteen days after bone marrow transplantation, both chimeric and control mice had normal platelet counts, but chimeric mice had no Rap1b detectable by Western blotting of platelet lysates with an anti-Rap1b antibody (data not shown).
The tail bleeding time of control mice engrafted with normal bone marrow was $142.6 \pm 44.5$ seconds (mean \pm SEM, Figure $2 \mathrm{C}$ ), a small but not statistically significant $(P<0.2)$ increase from that observed in normal mice (Figure 2A). In contrast, Rap1bnull bone marrow chimeras had a prolonged bleeding time of $396.0 \pm 89.9$ seconds (mean \pm SEM, Figure $2 \mathrm{D}$ ), which was similar to that of Rap1b-null mice (Figure 2B). These findings demonstrate that the deficiency of Rap $1 \mathrm{~b}$ in platelets prolongs bleeding time and that the abnormal platelet function in Rap1b-null mice results in a hemostatic defect.

Platelet aggregation is reduced in Rap $1 b$-null platelets. To characterize the platelet defects responsible for impaired hemostasis in Rap1bnull mice, we compared aggregation of Rap1b-null platelets with that of normal platelets in response to several mechanistically different agonists (Figure 3). The extent of aggregation of Rap1b-null platelets in response to ADP (Figure 3A) and epinephrine (Figure $3 \mathrm{~B})$, both weak agonists, was reduced over a wide range of agonist concentration (35-60\% and $20-40 \%$, respectively, compared with that in normal platelets).

Type I collagen, a strong platelet agonist, elicited cell shape changes reflected as an initial decrease in light transmission, followed by $65 \%$ maximal aggregation, when used at a concentration of $1.5-2 \mu \mathrm{g} / \mathrm{ml}$ in normal platelets (Figure 3C). The same concentration range of collagen was ineffective at inducing aggregation in Rap1b-null platelets, and only a small decrease in light transmission was recorded, indicating that platelet shape change had commenced. In addition, low concentrations of collagen induced a lower level of ATP secretion in Rap1b-null platelets as compared with normal platelets (data not shown). This reduced response was overcome with higher concentrations of collagen (at and above $5 \mu \mathrm{g} / \mathrm{ml}$ ), leading to the same extent of aggregation by Rap1b-null platelets and normal platelets. However, even at the highest concentrations of collagen tested, the shape change was slower and the time to half-maximal aggregation was increased in Rap1b-null cells.

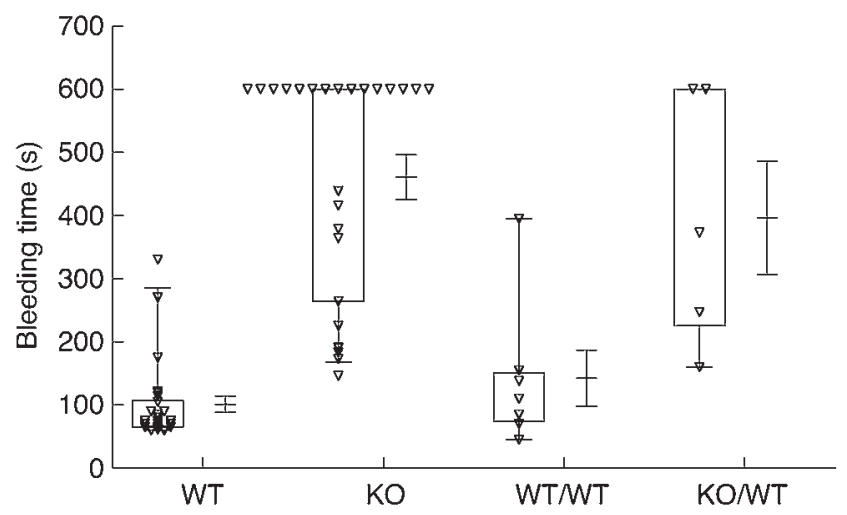

\section{Figure 2}

Prolonged tail bleeding time in Rap1b-null mice and in Rap1b-null bone marrow chimeras. Plotted are data from normal mice and bone marrow recipients with normal platelet counts in which the appropriate phenotype of the platelets was confirmed by Western blotting analysis. For each data set, the box shows the 25th to 75th percentile range and median, which in the KO group overlaps with the 75th percentile. Whiskers extend to the 5th and 95th percentiles and mean and SEM are plotted to the right of the box. WT/WT, chimeric normal mice transplanted with normal bone marrow; KO/WT, chimeric normal mice transplanted with Rap1b-null bone marrow. 


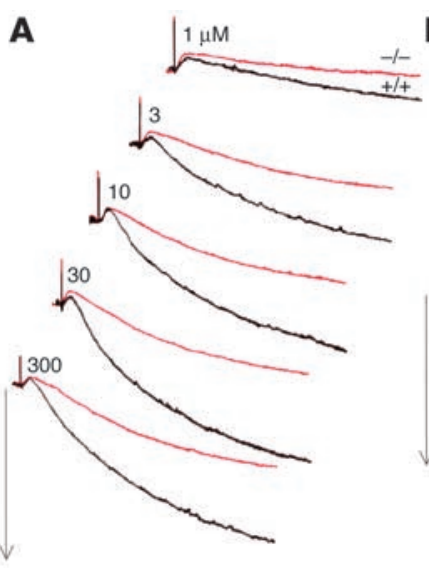

\section{B}
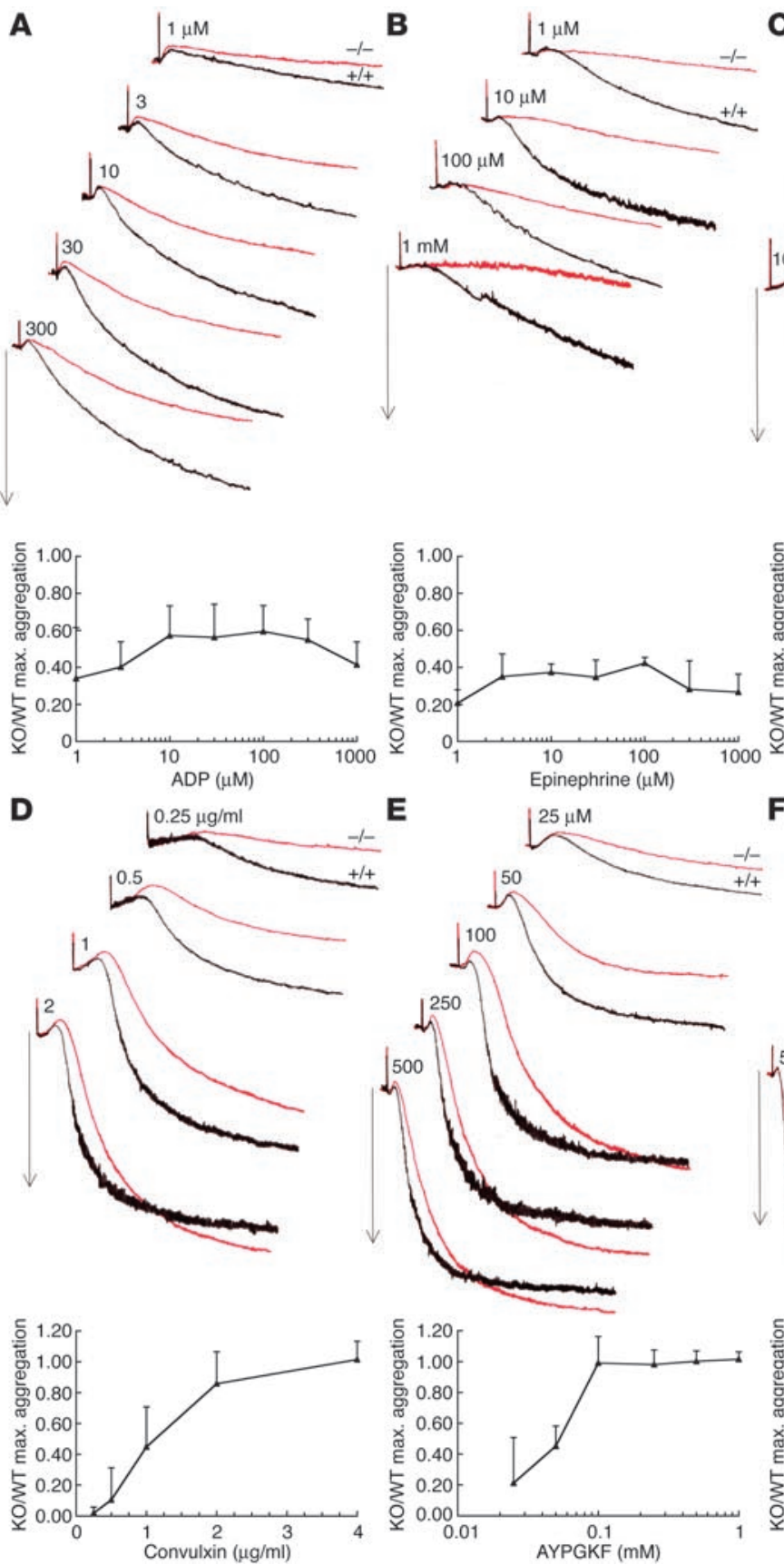

Convulxin, a snake venom C-type lectin (23), binds to glycoprotein GPVI (GPVI) and induces platelet activation by clustering the receptor. Unlike collagen, convulxin-induced activation of platelets is ADP-independent and occurs in the presence of ADP receptor antagonists and ADP scavengers (24). Despite this difference in the signaling pathway triggered, convulxin elicited a similar response in Rap1b-null platelets to that observed with collagen: a reduction in the extent of aggregation was most pronounced at low doses of convulxin, but decreased kinetics of aggregation were apparent even at highest concentration of the agonist (Figure 3D).

Stimulation of the $\mathrm{G} \alpha_{\mathrm{q}}$-linked PAR4 receptor activates platelets by triggering PLC $\beta$ activation, phosphoinosite hydrolysis, and intracellular calcium increase (25), providing a necessary and suf-

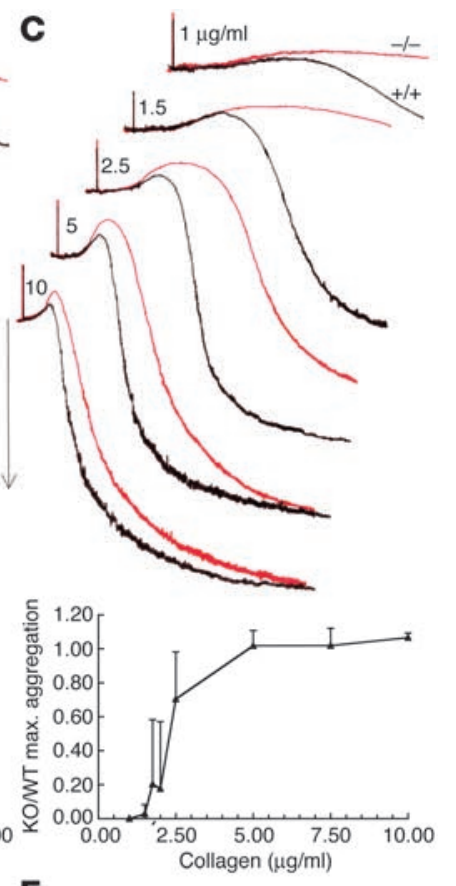

Figure 3

Inhibition of aggregation responses in Rap1b-null platelets. Typical aggregation traces of wild-type $(+/+$, black lines) and Rap1b-null $(-/-$, red lines) washed platelets in response to the following agonists: (A) ADP, (B) epinephrine, (C) type-I collagen, (D) convulxin, (E) AYPGKF peptide, and (F) calcium ionophore A23187. Arrows indicate $50 \%$ light transmission. Graphs represent the average maximum (max.) aggregation of rap1b-null platelets relative to that of normal platelets at a given agonist concentration. Error bars represent $\operatorname{SD}(n \geq 4)$.
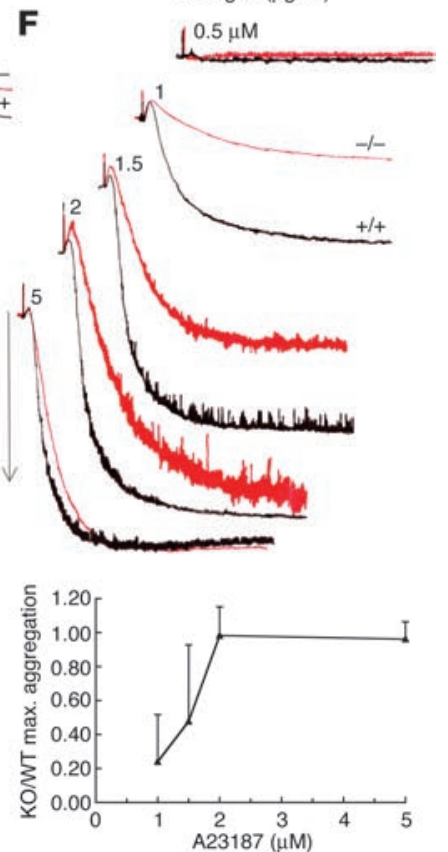

ficient stimulus for rapid Rap1b activation (13). Stimulation of Rap1b-null platelets with the PAR4 receptor activating peptide AYPGKF (Figure 3E) led to a right-shifted dose-response curve compared with that of normal platelets, similar to the aggregation defect observed with type I collagen and convulxin. Again, as with collagen and convulxin, shape change was slower and the time taken to reach half-maximal aggregation was increased in Rap1bnull platelets, even at the highest peptide concentration tested.

Treatment of platelets with calcium ionophore $\mathrm{A} 23187$ bypasses G protein-coupled receptors (GPCRs) and directly raises intracellular calcium. In response to this agonist, the extent of aggregation observed in Rap1b-null platelets was decreased by $50-75 \%$ in response to A23187 concentrations below $2 \mu \mathrm{M}$ 
A
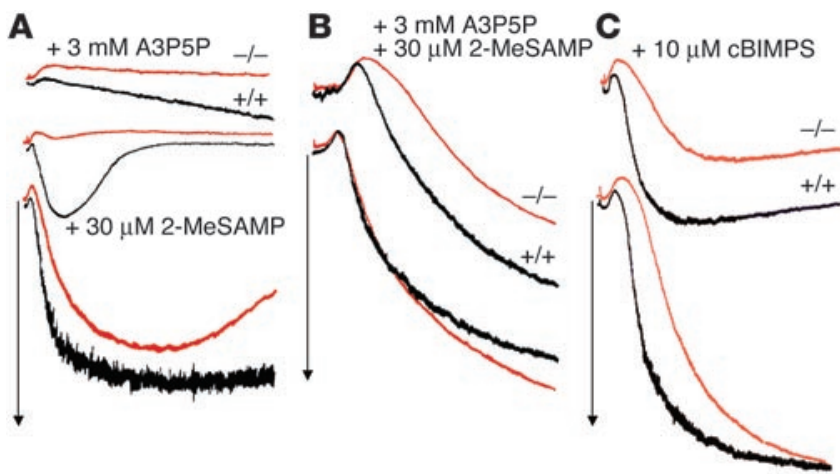

Figure 4

Rap1b-null platelets are susceptible to inhibition by purinergic receptor antagonists and CAMP. Typical aggregation traces of wild-type (+/+, black lines) and Rap1b-null (-/-, red lines) washed platelets and $\mathrm{PRP}$ in response to agonists in the absence and presence of indicated inhibitors. (A) PRP response to $5 \mu \mathrm{M}$ ADP. (B) Aggregation response of washed platelets to $50 \mu \mathrm{g} / \mathrm{ml}$ collagen. (C) Aggregation response of washed platelets to $0.1 \mathrm{mM}$ AYPGKF peptide. Arrows indicate $50 \%$ light transmission. $n=3$. 2-MeSAMP, 2-methylthio-AMP; A3P5P, adenosine 3'-5'-diphosphate; cBIMPS, Sp-5,6-DCL-cBIMPS.

(Figure 3F). Again, higher concentrations of A23187 elicited the same extent of aggregation in Rap1b-null as in normal platelets; however, the time taken to reach half-maximal aggregation was increased. Compared with normal platelets, Rap1b-null platelets elicited a right-shifted dose-response curve to A23187, with increased time to half-maximal aggregation and slower shape change even at the highest concentration tested.

Rap1b-null platelets are susceptible to inbibition by both purinergic receptor antagonists. Platelet aggregation in response to ADP depends on signaling from 2 GPCR-linked ADP receptors: a $\mathrm{G}_{\mathrm{q}^{-}}$ coupled $\mathrm{P}_{2} \mathrm{Y}_{1}$ receptor, responsible for platelet shape change and calcium mobilization, and a $\mathrm{G}_{\mathrm{i}}$-coupled $\mathrm{P} 2 \mathrm{Y}_{12}$ receptor, involved in the inhibition of adenylyl cyclase and stabilization of platelet aggregation. In response to ADP, the major pathway for Rap1b activation has been proposed to be downstream from the $\mathrm{P} 2 \mathrm{Y}_{12}$ receptor, with the $\mathrm{P} 2 \mathrm{Y}_{1}$ receptor playing a minor role (17). To investigate further whether Rap1b deficiency alters one or both purinergic receptor signaling pathways, we examined aggregation of Rap1b-null platelet-rich plasma (PRP) in response to ADP in the presence of specific purinergic receptor antagonists. In the presence of 2-methylthio-AMP (2-MeSAMP), a specific antagonist of the $\mathrm{P}_{2} \mathrm{Y}_{12}$ receptor, the extent of aggregation of wild-type platelets was inhibited and aggregation was completely reversed within 5 minutes. Under the same conditions, aggregation of Rap1b-null platelets was completely blocked (Figure 4A). The $\mathrm{P}_{2} \mathrm{Y}_{1}$ receptor antagonist adenosine $3^{\prime}-5^{\prime}$-diphosphate (A3P5P) completely obliterated the response of Rap1b-null PRP to ADP and almost completely inhibited the response of normal platelets (Figure 4A). Thus, Rap1b-null platelets are susceptible to antagonists of both purinergic receptors, indicating that the absence of Rap1b does not completely block signaling from either receptor. To further investigate the effect of decreased ADP signaling on the decreased aggregation of Rap1b-null platelets in response to other agonists, we tested the effect of combined purinergic receptor antagonists on collagen-induced aggregation (Figure 4B). Obliteration of signaling by secreted ADP had a modest effect on the aggregation of normal platelets in response to high concentration of collagen; however, aggregation of Rap1b-null platelets was inhibited to a larger extent, indicating that Rap1b deficiency interferes with signaling downstream from GPVI, the main collagen receptor involved in platelet activation (24). Similar data was obtained with PAR4 activating peptide (data not shown).

Rap1b-null platelets are susceptible to inhibition by cAMP. Elevation of cAMP concentration in platelets leads to the inhibition of agonist-induced platelet activation and is mediated, in part, by cAMP-dependent protein kinase (PKA). Because Rap1b is one of the proteins that become phosphorylated by PKA upon elevation of intracellular cAMP levels, we evaluated the effect of the absence of Rap1b in platelets on cAMP-induced inhibition of AYPGKF peptide-induced platelet aggregation (Figure 4C). Pretreatment of normal platelets with a membrane-permeable analog of cAMP, Sp-5,6-DCL-cBIMPS, dose-dependently inhibited AYPGKF-induced
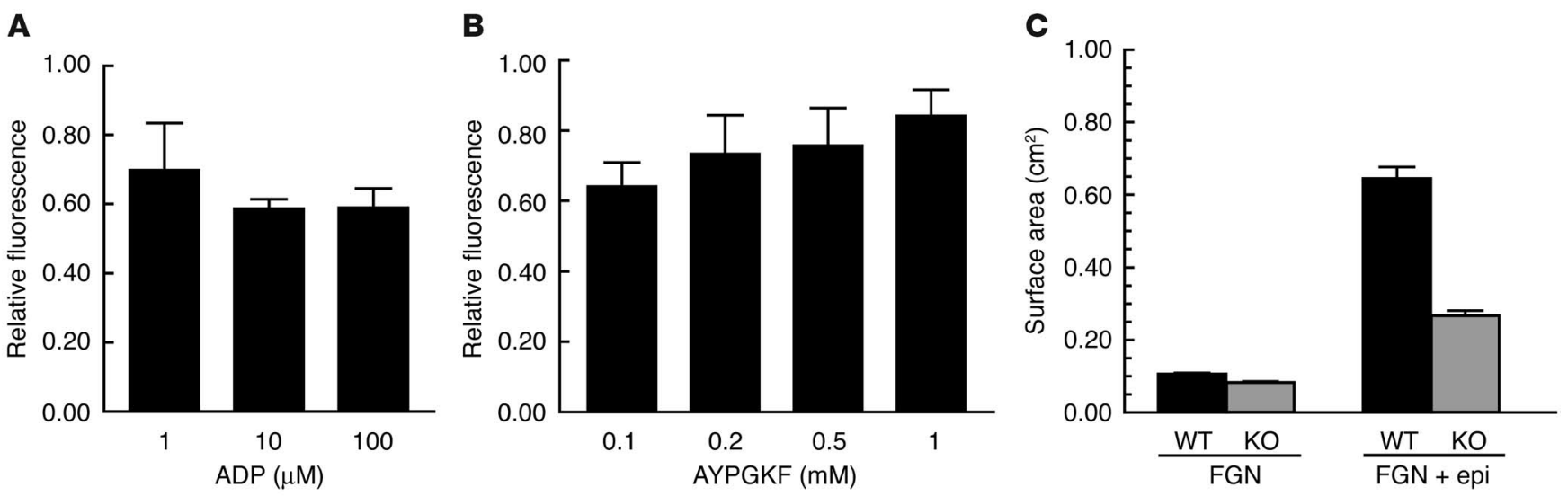

Figure 5

Impaired soluble and solid-phase fibrinogen binding to Rap1b-null platelets. (A) Specific soluble fibrinogen binding to Rap1b-null platelets, presented relative to that of normal platelets, arbitrarily set at $100 \%$, in the presence of the indicated concentrations of ADP. (B) Specific soluble fibrinogen binding to Rap1b-null platelets, relative to that of normal platelets, in the presence of AYPGKF peptide. (C) Wild-type and Rap1b-null platelet spreading on fibrinogen-coated coverslips in the absence (FGN) or presence (FGN + epi) of epinephrine. Data represent mean \pm SEM $(n>30) . P<0.0001$ by $t$ test compared with control. 

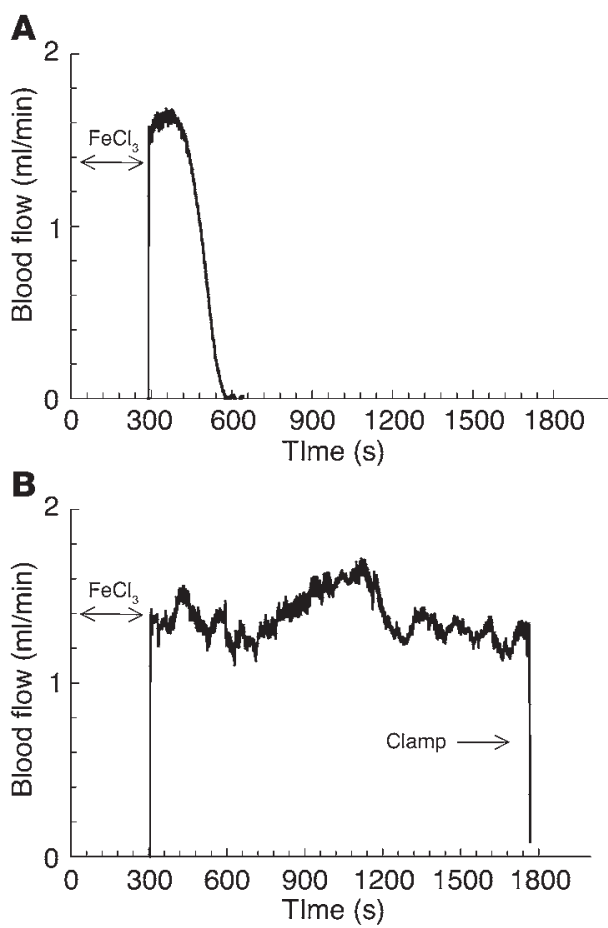

\section{Figure 6}

Inhibition of arterial thrombus formation in rap1b-deficient mice. Blood flow through the carotid artery monitored immediately before and after $\mathrm{FeCl}_{3}$ injury to the artery, represented as time 0 . (A) Blood flow trace of a wild-type mouse. (B) Blood flow trace of a Rap1b-null mouse. Time of occlusion in the control mice was $7.0 \pm 1.30$ minutes (mean $\pm S D$; $n=6$ ). The blood flow did not stop in the Rap1b-null mice throughout the time of experiment $(n=6)$.

aggregation in both normal and Rap1b-null platelets. The extent of inhibition was similar in normal and Rap1b-null platelets activated by AYPGKF peptide. Similar results were obtained when ADP or type I collagen were used as agonists (data not shown). Thus, Rap1b is not required for cAMP inhibition of platelet aggregation.

Rap1b deficiency impairs integrin $\alpha_{I i b} \beta_{3}$ activation and downstream signaling. To investigate the effect of Rap1b deficiency on agonist-induced, "inside-out" integrin $\alpha_{\mathrm{II}} \beta_{3}$ activation, we compared the ability of Rap1b-null and normal platelets to bind soluble fibrinogen upon stimulation with a range of concentrations of ADP or PAR4 activating peptide AYPGKF. ADP-stimulated fibrinogen binding was 30-40\% lower in Rap1b-null platelets compared with wild-type platelets (Figure 5A). AYPGKF peptide, a strong agonist, stimulated a higher level of fibrinogen binding to normal platelets than ADP (data not shown), but binding was still significantly reduced in Rap1b-null platelets (Figure 5B). To exclude the possibility that the decreased level of fibrinogen binding in Rap1b-null platelets might be due to decreased expression of the fibrinogen receptor, we quantified surface expression of integrin $\alpha_{\mathrm{II}} \beta_{3}$ using flow cytometric analysis of anti-mouse $\alpha_{\text {IIb }}$ (CD41) antibody binding and found that surface expression of immunoreactive integrin $\alpha_{\mathrm{IIb}} \beta_{3}$ in Rap1b-null platelets was similar to that in wild-type platelets (data not shown).

Next, we compared the spreading of Rap1b-null and normal platelets on solid-phase fibrinogen, a process that depends on integrin-dependent reorganization of the cytoskeleton. Without prior stimulation, normal murine platelets spread only to a limited extent; nevertheless, even this limited spreading was inhibited in Rap1b-null platelets (Figure 5C). Pretreatment of platelets with epinephrine enhanced spreading of both normal and Rap1b-null platelets; however, the surface area of Rap1b-null platelets was decreased by $55 \%$ compared with that of normal platelets. Therefore, Rap $1 b$ deficiency inhibits both integrin activation and processes downstream from the integrins.

Rap1b-null mice are protected from thrombosis in an in vivo thrombosis model. To examine the effect of Rap1b deficiency on platelet function in vivo, we used a platelet-dependent carotid artery thrombosis model (26) that requires integrin $\alpha_{\mathrm{IIb}} \beta_{3}$ (27). In normal mice, the average time to vessel occlusion was $7.0 \pm 1.30$ minutes (mean \pm SD, $n=6$ ) (Figure 6A). Strikingly, none of the 6 Rap $1 \mathrm{~b}$-null mice tested thrombosed in the 30 minutes of observation (Figure 6B). Microscopic evaluation of carotid arteries from Rap1b-null mice revealed that a thrombus had began to form but did not occlude the vessel (data not shown).

\section{Discussion}

The results of this study demonstrate that Rap1b-null mice have a bleeding defect due to abnormal platelet function. In vitro aggregation responses of Rap1b-null platelets are impaired, and this impairment appears to be due to impaired activity of integrin. Moreover, in an in vivo, platelet-dependent arterial thrombosis model, in which integrin $\alpha_{I I b} \beta_{3}$ is a central regulator, Rap1b-null platelets are protected from thrombosis.

In contrast to previous studies that have placed Rap1b in the signaling pathway downstream from the $\mathrm{G}_{\mathrm{i}}$-coupled receptors (16-18), the data presented in this paper suggest that Rap1b is involved in a common critical step required for platelet activation. Consistent with the former model and similar to the $\mathrm{Ga}_{\mathrm{i} 2}$-null phenotype (28), ADP and epinephrine-induced aggregation is attenuated in Rap1b-null platelets. Rap1b-null platelet aggregation is also dramatically reduced at lower collagen concentrations, where normal platelet responses are dependent on secreted ADP and integrin $\alpha_{\mathrm{IIb}} \beta_{3}$ signaling (29). However, ADP-induced aggregation of Rap1b-null platelets is further inhibited when signaling from either of the purinergic receptors is blocked with receptor-specific antagonists, which argues against Rap1b functioning exclusively in one of those pathways. Moreover, early signaling events from the $\mathrm{P} 2 \mathrm{Y}_{1}$ and $\mathrm{P} 2 \mathrm{Y}_{12}$ receptors (the increase in cytosolic calcium and the inhibition of adenylyl cyclase, respectively) are not affected in Rap1b-null platelets (data not shown), indicating that Rap1b lies further downstream from these events.

Strikingly, aggregation of Rap1b-null platelets is impaired in response to all agonists tested, not just those coupled to GPCRs. Dose-response curves to the $\mathrm{G}_{\mathrm{q}}$-coupled PAR4 agonist peptide or non-GPCR-coupled GPVI receptor agonists (convulxin and high concentration of collagen, which is ADP-independent; ref. 30) are all right-shifted in Rap1b-null platelets relative to normal platelets. Moreover, inhibition of the effects of released ADP by the use of purinergic receptor antagonists further reduces aggregation of Rap1-null platelets. The abnormalities in Rap1b-null platelets are not overcome by higher doses of agonist: the slower shape change and reduced rate of aggregation persist. Additionally, Rap1b-null platelets manifest similar defects in response to calcium ionophore, indicating that Rap1b acts downstream from calcium release.

One critical step in platelet activation regulated by Rap1b is integrin $\alpha_{\mathrm{II}} \beta_{3}$ activation. We show that Rap1b deficiency impairs both weak and strong agonist-induced soluble fibrinogen binding, indicating that Rap $1 \mathrm{~b}$ is involved upstream from integrin $\alpha_{\mathrm{IIb}} \beta_{3}$ activation. In addition, platelet spreading on fibrinogen, a complex 
phenomenon dependent on the signaling downstream from integrin engagement, is decreased in Rap1b-null platelets. Our thrombosis model results support the idea that Rap $1 \mathrm{~b}$ is a critical regulator of integrin function in vivo.

While the exact mechanism through which Rap $1 \mathrm{~b}$ regulates integrin activity remains unknown, several of our results and previous reports suggest that Rap1b may be acting between integrin $\alpha_{\text {IIb }} \beta_{3}$ and the cytoskeleton. A universal characteristic in all Rap1b-null platelet aggregations is slower shape change. This could result from slower integrin activation and/or decreased actin microfilament dynamics. This phenotype is opposite to that seen in another knockout model of a platelet-abundant protein, vasodilator-stimulated phosphoprotein (VASP), which is believed to negatively regulate actin polymerization. In VASP-null platelets, collagen induces faster shape change (31) and increased fibrinogen binding compared with normal platelets $(31,32)$. Moreover, VASP, which, like Rap1b, is phosphorylated by PKA upon elevation of cellular cAMP level, has been shown to be a mediator of that inhibition (31). Unlike VASP-null platelets, aggregation of Rap1b-null platelets is susceptible to inhibition by a full range of cAMP concentrations, indicating that Rap $1 \mathrm{~b}$ is not a critical effector of cAMP inhibition.

Another indication that Rap1b may be involved in regulating cytoskeleton-integrin interaction comes from localization studies. Upon stimulation and aggregation of platelets, Rap1b translocates to the cytoskeleton $(33,34)$ in a process that is largely integrinindependent, as it occurs in platelets from Glanzmann thrombesthenia patients (35). Lastly, studies in megakaryocytes have shown that Rap1b-induced upregulation of integrin is blocked when actin polymerization is blocked by cytochalasin $\mathrm{D}(20)$.

This study shows that Rap $1 \mathrm{~b}$ is involved in regulation of platelet function and that its deficiency leads to a mild bleeding defect in otherwise apparently normal mice. However, the mildness of the defect in surviving adults contrasts with a severe bleeding phenotype and lethality of Rap1b-null embryos. While the surviving Rap1b-null mice may have a genotypic or phenotypic compensation that ameliorates the consequences of rap1b deletion, we believe that the defective function of Rap1b-null platelets is unlikely to be the cause of increased embryonic lethality. In fact, neither platelets nor fibrinogen are required for embryonic hemostasis $(36,37)$. Instead, embryonic bleeding is often caused by a defective vascular component of hemostasis. Whether Rap $1 \mathrm{~b}$ is involved in the embryonic vasculogenesis is an intriguing question.

Interestingly, Rap1a, which is highly homologous to Rap1b and is also expressed in platelets, albeit at a lower level (12), does not appear to be required for platelet function, as Rap1a-null platelets have normal aggregation responses (M. Chrzanowska-Wodnicka and L.A. Quilliam, unpublished data). Moreover, the activity of Rap1a is undetectable in Rap1b-deficient platelets (M.K. Larson, M. Chrzanowska-Wodnicka, G.C. White II, and L.V. Parise, unpublished observations), and thus, Rap1a does not appear to compensate for Rap1b deficiency in platelets.

This report provides the first genetic evidence to our knowledge that Rap $1 \mathrm{~b}$ is required for normal integrin $\alpha_{\mathrm{II}} \beta_{3}$ signaling in platelets. Impaired integrin signaling in Rap1b-null platelets is likely to be a major factor responsible for protection from thrombosis in Rap1b-null mice in the in vivo arterial thrombosis model, where integrin $\alpha_{\text {IIb }} \beta_{3}$ is key (27). Our study shows that Rap1b is required for normal hemostasis in vivo and, because its deficiency confers protection against thrombosis without spontaneous bleeding, Rap1b emerges as a novel antithrombotic therapy target.
The next challenge will be to elucidate the exact mechanisms by which Rap1b regulates signaling from the receptor agonists to integrin $\alpha_{\text {IIb }} \beta_{3}$, affecting platelet function. The Rap1b-knockout mice should provide a useful tool to accomplish that goal.

\section{Methods}

Materials. All reagents were from Sigma-Aldrich unless indicated otherwise, including type I bovine collagen (Chrono-Log Corp.), convulxin (Pentapharm), and PAR4 agonist peptide (AYPGKF), which was synthesized and HPLC-purified at the UNC Microprotein Sequencing and Peptide Synthesis Facility. rap1b gene targeting in embryonic stem cells (ES cells) was performed by the Animal Models Core Facility at the University of North Carolina at Chapel Hill.

Generation of Rap1b-null mice. All animal and recombinant DNA experiments were approved by the Institutional Animal Care and Use Committee of the University of North Carolina at Chapel Hill. A 129 Sv/Ev mouse genomic library in lambda FIX phage (Stratagene) was screened with a full-length rap $1 \mathrm{~b}$ cDNA probe. A positive clone containing the entire rap $1 b$ coding sequence was used as a template for a series of PCR reactions using the primers located in the phage arm T7 and T3 sites and encompassing codons $18-12$ and 172 and 180 of rap $1 \mathrm{~b}$ cDNA, respectively, to yield $3.3-\mathrm{kb}$ and 7.2-kb PCR products, respectively, which were then subcloned into pCRII vector and sequenced to confirm the identity of the clone as rap1b. The rap $1 b$ replacement vector was constructed by flanking the selection cassette containing the neomycin-resistance gene with poly(A) sequences under the control of the PGK1 promoter of $\mathrm{PJNS}_{5}$ vector (38) with $1.7-\mathrm{kb}$ and $6-\mathrm{kb}$ portions of genomic DNA flanking the rap 16 open reading frame. The vector, linearized with NotI, was electroporated into $129 \mathrm{~Sv} / \mathrm{Ev}$ ES cells, and neomycin-resistant clones that survived negative selection by gancyclovir were screened by PCR using primers located in the $5^{\prime}$ UTR (5'-CATCTTCAGTCCCCAATGGGC-3') and in the $3^{\prime}$ end of the neomycinresistance gene (5'-TGCTCCAGACTGCCTTGG-3'). PCR-positive clones were genotyped using Southern blotting; genomic DNA was digested with SspI and KpnI and probed with a 270-bp NotI-HindIII fragment from the rap $1 b$ coding sequence outside of the upstream arm. Mutant ES cell clones were injected into C57BL/6 blastocysts to make chimeras, which were subsequently mated with C57BL/6 mice. Germline transmission was confirmed by PCR and Southern blot analysis. Heterozygous animals were intercrossed to obtain $\mathrm{rap} 1 \mathrm{~b}^{-/-}$mice. The absence of the rap $1 b$ gene product in platelets and other tissues (data not shown) was confirmed by Western blotting of cell lysates with an anti-Rap1b antibody, while a parallel blot with an anti-Rap1a-specific antibody did not show any increase in the Rap1a expression in Rap1b-null platelets. E15.5 embryos were obtained from staged heterozygous intercrosses, visualized with a Leica M420 Macroscope, and euthanized, and tissue was harvested for genotyping.

Bone marrow transplantation. Bone marrow was obtained from femurs and tibias of 1-month-old normal and Rap1b-null mice and resuspended in RPMI 1640 medium at $1 \times 10^{7}$ cells $/ \mathrm{ml}$. The bone marrow cell suspension $(0.2 \mathrm{ml})$ was injected into the tail veins of lethally irradiated normal mice. After 14 days, tail bleeding assays, automated blood platelet counts, and Western blot analysis of platelet Rap1b expression using goat anti-Rap1b polyclonal antibody (Santa Cruz Biotechnology Inc.) were performed.

Tail bleeding assays. Tail bleeding assays were performed in 5- to 6-week-old

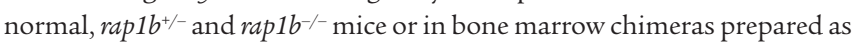
described above, following a previously published protocol (39). Briefly, $3 \mathrm{~mm}$ of the distal tip of the tails were cut from restrained mice and immediately immersed in $0.9 \%$ isotonic saline at $37^{\circ} \mathrm{C}$. Tail bleeding time was defined as time required for bleeding to stop. In cases where bleeding did not stop spontaneously at 10 minutes, bleeding was stopped at that time by cauterizing the tail and bleeding time was indicated as 10 minutes. 
Platelet preparation. Mouse platelets were obtained using a modified protocol of Jantzen et al. (28) as follows. Blood was acquired by cardiac puncture into $10 \%$ volume of HEPES/Tyrodes buffer $\mathrm{pH} 7.4$ (10 mM HEPES, $12 \mathrm{mM} \mathrm{NaHCO}_{3}, 138 \mathrm{mM} \mathrm{NaCl}, 5.5 \mathrm{mM}$ glucose, and $2.9 \mathrm{mM} \mathrm{KCl}$ ) containing $15 \mathrm{U} / \mathrm{ml}$ heparin and centrifuged at $86 \mathrm{~g}$ for 8 minutes to collect PRP. To increase platelet yield, the lower phase was washed 3 times with HEPES/Tyrodes buffer (with $10 \mathrm{mM}$ EDTA and $1 \mu \mathrm{M}$ prostaglandin $\mathrm{E}_{1}$ added to first wash only) and obtained platelet-rich buffers were combined. Red blood cells remaining in the PRP were pelleted at $500 \mathrm{~g}$ for 30 seconds, and platelets were collected from the suspension by centrifugation at $718 \mathrm{~g}$ for 6 minutes. The platelet pellet was resuspended in $1 \mathrm{ml}$ of CGS $(13 \mathrm{mM}$ sodium citrate, $30 \mathrm{mM}$ glucose, and $120 \mathrm{mM} \mathrm{NaCl}$ ) with $1 \mathrm{U} / \mathrm{ml}$ apyrase (grade VII) followed by 15 minutes of incubation at $37^{\circ} \mathrm{C}$. Platelets were collected and resuspended at $2 \times 10^{8} / \mathrm{ml}$ in HEPES/Tyrodes buffer with $1 \mathrm{mM} \mathrm{CaCl}_{2}$ and allowed to rest for 30 minutes at room temperature. For analysis of purinergic receptor antagonists on ADP-induced aggregation, enoxoparin, a low-molecular-weight heparin (Lovenox, Aventis), was used as an anticoagulant, and PRP was collected after addition of $100 \mu \mathrm{l}$ of PBS to the whole blood. Platelet count was adjusted to $2 \times 10^{8} / \mathrm{ml}$.

Platelet aggregations. Aggregation of washed and rested platelets was performed in an aggregometer (Chrono-Log Corp.) with stirring at $37^{\circ} \mathrm{C}$ in the presence of $0.4 \mathrm{mg} / \mathrm{ml}$ of human fibronectin-depleted fibrinogen (Enzyme Research Laboratories) and agonists and inhibitors as described in Results. The extent of aggregation was determined after 5 minutes from the addition of the agonist and expressed as a percent of maximum aggregation. For each concentration of agonist, an average percent aggregation and error were calculated as a mean $\pm S D$, respectively, of at least 4 experiments.

Fibrinogen binding assay and FACS analysis. Washed, rested platelets were incubated for 20 minutes at room temperature with $250-500 \mu \mathrm{g} / \mathrm{ml}$ of biotin-fibrinogen and ADP or PAR4 agonist peptide in $50 \mu \mathrm{l}$ final volume of HEPES/Tyrodes buffer with $1 \mathrm{mM} \mathrm{CaCl}_{2}$, in the presence of indicated agonists and 1-4 $\mu$ l of phycoerythrin-streptavidin (Jackson ImmunoResearch Laboratories Inc.). Surface expression of integrin $\alpha_{\text {IIb }} \beta_{3}$ on wild-type and Rap1b-null platelets was measured by flow cytometry after staining with a FITC-labeled anti-murine $\alpha_{\text {IIb }}$ antibody or isotype-matched control (BD Biosciences - Pharmingen).

Platelet spreading on solid-phase fibrinogen. Glass coverslips were coated with $50 \mu \mathrm{g} / \mathrm{ml}$ of human fibrinogen in HEPES/Tyrodes buffer for 2 hours at room temperature, and nonspecific binding sites were blocked with $0.5 \%$ bovine serum albumin. Platelets, at $3 \times 10^{7}$ cells $/ \mathrm{ml}$, were allowed to spread on coverslips for 45 minutes at $37^{\circ} \mathrm{C}$ in the absence or presence of $50 \mu \mathrm{M}$ epinephrine and then were fixed and mounted onto glass slides. The degree of spreading was quantified as cell surface area on phase contrast photographs using computer-assisted analysis (MCID software). The experiments were performed using at least 2 individual sets of wild-type and Rap1b-null mice.

In vivo thrombosis model. Carotid artery thrombosis studies were performed in 8- to 12-week-old mice as described (26). Blood flow in the right common carotid artery and thrombus formation were evaluated for 30 minutes after the injury was induced with $20 \% \mathrm{FeCl}_{3}$. Time to occlusion was defined as time from the injury until blood flow was reduced to less than $10 \%$ of the original flow for at least 30 seconds. To evaluate thrombus formation microscopically, the carotid arteries were fixed in $4 \%$ paraformaldehyde in PBS and embedded, sectioned, and stained with hematoxylin-eosin as described before (27).

\section{Acknowledgments}

We would like to thank our colleagues from the University of North Carolina at Chapel Hill: Randy Thresher (Animal Models Core Facility) for his help with the targeting vector, Jennifer Cullen, Anna Kraus and Eleanor Hilliard for excellent technical assistance, Mauricio Rojas and Zehra Pamuklar for performing in vitro thrombosis experiments, Sheritha Lee for help with the bone marrow transplants experiments, and Zenon Rajfur for help with imaging. We would also like to thank Sandy Shattil, Shaun Jackson, and Lawrence Brass for stimulating discussions. This work was supported by AHA Scientist Development grant 0235127N (to M. Chrzanowska-Wodnicka), NIH grant HL-45100 (to G.C. White), and National Heart Foundation of Australia grant G 01M 0330 and NHMRC 194205 (to S.M. Schoenwaelder). S.M. Schoenwaelder is a recipient of NHMRC RD Wright Fellowship and Monash University Logan fellowship.

Received for publication August 9, 2004, and accepted in revised form December 14, 2004.

Address correspondence to: Magdalena Chrzanowska-Wodnicka, Department of Medicine, University of North Carolina at Chapel Hill, 932 Mary Ellen Jones Building, Chapel Hill, North Carolina 27599, USA. Phone: (919) 966-3769; Fax: (919) 966-7639; E-mail: Magdalena_Wodnicka@med.unc.edu.
1. Robbins, S.M., Suttorp, V.V., Weeks, G., and Spiegelman, G.B. 1990. A ras-related gene from the lower eukaryote Dictyostelium that is highly conserved relative to the human rap genes. Nucleic Acids Res. 18:5265-5269.

2. Robbins, S., Khosla, M., Thiery, R., Weeks, G., and Spiegelman, G.B. 1991. Ras-related genes in Dictyostelium discoideum. Dev. Genet. 12:147-153.

3. Hariharan, I.K., Carthew, R.W., and Rubin, G.M. 1991. The Drosophila roughened mutation: activation of a rap homolog disrupts eye development and interferes with cell determination. Cell. 67:717-722.

4. Pizon, V., Lerosey, I., Chardin, P., and Tavitian, A. 1988. Nucleotide sequence of a human cDNA encoding a ras-related protein (rap1B). Nucleic Acids Res. 16:7719.

5. Kang, R., Kae, H., Ip, H., Spiegelman, G.B., and Weeks, G. 2002. Evidence for a role for the Dictyostelium Rap1 in cell viability and the response to osmotic stress. J. Cell Sci. 115:3675-3682.

6. Kitayama, H. 1989. A ras-related gene with transformation suppressor activity. Cell. 56:77-84.

7. Campa, M.J., Chang, K.J., Molina y Vedia, L., Reep, B.R., and Lapetina, E.G. 1991. Inhibition of ras- induced germinal vesicle breakdown in Xenopus oocytes by rap-1B. Biochem. Biophys. Res. Commun. 174:1-5.

8. Knox, A.L., and Brown, N.H. 2002. Rap1 GTPase regulation of adherens junction positioning and cell adhesion. Science. 295:1285-1288.

9. Caron, E. 2003. Cellular functions of the Rap1 GTP-binding protein: a pattern emerges. J. Cell Sci. 116:435-440.

10. Bos, J.L., et al. 2003. The role of Rap1 in integrin-mediated cell adhesion. Biochem. Soc. Trans. 31:83-86.

11. Stork, P.J. 2003. Does Rap1 deserve a bad Rap? Trends Biochem. Sci. 28:267-275.

12. Klinz, F.J., et al. 1992. Generation of specific antibodies against the rap1A, rap1B and rap2 small GTPbinding proteins. Analysis of rap and ras proteins in membranes from mammalian cells. Eur. J. Biochem. 207:207-213.

13. Franke, B., Akkerman, J.-W.N., and Bos, J.L. 1997. Rapid Ca2+-mediated activation of Rap1 in human platelets. EMBO J. 16:252-259.

14. Zwartkruis, F.J., Wolthuis, R.M., Nabben, N.M., Franke, B., and Bos, J.L. 1998. Extracellular signalregulated activation of Rap1 fails to interfere in Ras effector signalling. EMBO J. 17:5905-5912.

15. Franke, B., et al. 2000. Sequential regulation of the small GTPase Rap1 in human platelets. Mol. Cell. Biol. 20:779-785.

16. Lova, P., Paganini, S., Sinigaglia, F., Balduini, C., and Torti, M. 2002. A Gi-dependent pathway is required for activation of the small GTPase Rap1B in human platelets. J. Biol. Chem. 277:12009-12015.

17. Woulfe, D., Jiang, H., Mortensen, R., Yang, J., and Brass, L.F. 2002. Activation of Rap1B by Gi family members in platelets. J. Biol. Chem. 277:23382-23390.

18. Lova, P., et al. 2003. A selective role for phosphatidylinositol 3,4,5-trisphosphate in the Gi-dependent activation of platelet Rap1B. J. Biol. Chem. 278:131-138.

19. Eto, K., et al. 2002. Megakaryocytes derived from embryonic stem cells implicate CalDAG-GEFI in integrin signaling. Proc. Natl. Acad. Sci. U. S. A. 99:12819-12824.

20. Bertoni, A., et al. 2002. Relationships between Rap1b, affinity modulation of integrin alpha IIbbeta 3 , and the actin cytoskeleton. J. Biol. Chem. 277:25715-25721.

21. de Bruyn, K.M., et al. 2003. The small GTPase Rap 1 is activated by turbulence and is involved in integrin \{alpha\}IIb $\{$ beta\}3-mediated cell adhesion in human megakaryocytes. J. Biol. Chem. 
278:22412-22417.

22. Hodivala-Dilke, K.M., et al. 1999. Beta3-integrin-deficient mice are a model for Glanzmann thrombasthenia showing placental defects and reduced survival. J. Clin. Invest. 103:229-238.

23. Polgar, J., et al. 1997. Platelet activation and signal transduction by convulxin, a C-type lectin from crotalus durissus terrificus (tropical rattlesnake) venom via the p62/GPVI collagen receptor. J. Biol. Chem. 272:13576-13583.

24. Nieswandt, B., and Watson, S.P. 2003. Platelet-collagen interaction: is GPVI the central receptor? Blood. 102:449-461.

25. Brass, L.F. 2003. Thrombin and platelet activation. Chest. 124:18S-25S.

26. Jirouskova, M., Chereshnev, I., Vaananen, H., Degen, J.L., and Coller, B.S. 2004. Antibody blockade or mutation of the fibrinogen \{gamma\}-chain C-terminus is more effective in inhibiting murine arterial thrombus formation than complete absence of fibrinogen. Blood. 103:1995-2002.

27. Smyth, S.S., Reis, E.D., Vaananen, H., Zhang, W., and Coller, B.S. 2001. Variable protection of $\{$ beta\}3-integrin-deficient mice from thrombosis initiated by different mechanisms. Blood. 98:1055-1062.

28. Jantzen, H.-M., Milstone, D.S., Gousset, L., Conley,
P.B., and Mortensen, R.M. 2001. Impaired activation of murine platelets lacking G\{alpha\}i2. J. Clin. Invest. 108:477-483. doi:10.1172/JCI200112818.

29. Cho, M.J., et al. 2003. The roles of alpha IIbbeta 3-mediated outside-in signal transduction, thromboxane $\mathrm{A} 2$, and adenosine diphosphate in collagen-induced platelet aggregation. Blood. 101:2646-2651.

30. Quinton, T.M., Ozdener, F., Dangelmaier, C., Daniel, J.L., and Kunapuli, S.P. 2002. Glycoprotein VI-mediated platelet fibrinogen receptor activation occurs through calcium-sensitive and PKC-sensitive pathways without a requirement for secreted ADP. Blood. 99:3228-3234.

31. Aszodi, A., et al. 1999. The vasodilator-stimulated phosphoprotein (VASP) is involved in cGMP- and cAMP-mediated inhibition of agonist-induced platelet aggregation, but is dispensable for smooth muscle function. EMBO J. 18:37-48.

32. Hauser, W., et al. 1999. Megakaryocyte hyperplasia and enhanced agonist-induced platelet activation in vasodilator-stimulated phosphoprotein knockout mice. Proc. Natl. Acad. Sci. U. S. A. 96:8120-8125.

33. Fischer, T.H., Gatling, M.N., Lacal, J.-C., and White, G.C., 2nd. 1990. rap1B, a cAMP-dependent protein kinase substrate, associates with the platelet cytoskel- eton. J. Biol. Chem. 265:19405-19408.

34. Fischer, T.H., Gatling, M.N., McCormick, F., Duffy, C.M., and White, G.C., 2nd. 1994. Incorporation of Rap $1 \mathrm{~b}$ into the platelet cytoskeleton is dependent on thrombin activation and extracellular calcium. J. Biol. Chem. 269:17257-17261.

35. White, G.C., 2nd, Fisher, T.H., and Duffy, C.M. 1998. Rap1b association with the platelet cytoskeleton occurs in the absence of glycoproteins IIb/IIIa. Thromb. Haemost. 79:832-836.

36. Shivdasani, R.A., et al. 1995. Transcription factor NF-E2 is required for platelet formation independent of the actions of thrombopoietin/MGDF in megakaryocyte development. Cell. 81:695-704.

37. Suh, T.T., et al. 1995. Resolution of spontaneous bleeding events but failure of pregnancy in fibrinogen-deficient mice. Genes Dev. 9:2020-2033.

38. Tybulewicz, V.L., Crawford, C.E., Jackson, P.K., Bronson, R.T., and Mulligan, R.C. 1991. Neonatal lethality and lymphopenia in mice with a homozygous disruption of the c-abl proto-oncogene. Cell. 65:1153-1163.

39. Dejana, E., Quintana, A., Callioni, A., and de Gaetano, G. 1979. Bleeding time in laboratory animals. III Do tail bleeding times in rats only measure a platelet defect? (the aspirin puzzle). Thromb. Res. 15:199-207. 